\title{
Effect of mobile-based voice therapy on the voice quality of patients with dysphonia
}

\author{
Geun-Hyo Kim', Yeon-Woo Lee', In-Ho Bae², Hee-June Park³, Soon-Bok Kwon ${ }^{4}$ \\ ${ }^{1}$ Department of Otorhinolaryngology-Head and Neck Surgery and Biomedical Research Institute, Pusan National University Hospital, Busan; \\ ${ }^{2}$ Department of Speech-Language Pathology, Kosin University, Busan; ${ }^{3}$ Department of Speech and Hearing Therapy, Catholic University of Pusan, \\ Busan; ${ }^{4}$ Department of Humanities, Language and Information, Pusan National University, Busan, Korea
}

Purpose: Mobile-based voice therapy (MBVT) has the advantage of being cheaper and more flexible for patients and clinicians compared to traditional PC-based voice therapy. The purpose of this study was to investigate the effect of MBVT on the voice quality of patients with dysphonia.

Methods: Forty-five patients with benign vocal fold lesions were randomly allocated to either the experimental $(\mathrm{N}=25)$ or control $(\mathrm{N}=20)$ group. The experimental group received MBVT, while the control group received traditional voice therapy (TRVT). Both groups participated in 40 minutes of the intervention per session, once per week, for 8 weeks. Voice evaluation measures included cepstral analysis, acoustic voice quality index, acoustic breathiness index, auditory-perceptual ratings, and a self-rated questionnaire. Analyses compared the voice quality and subjective variables before and after each therapy, as well as between each type of therapy (MBVT vs. TRVT).

Results: The results showed that voice quality and patient satisfaction improved in both therapies compared to before therapy, indicating recovery $(p<0.01)$. Therefore, similar to TRVT, MBVT was effective for voice rehabilitation.

Conclusions: MBVT could have a positive effect on voice recovery for patients with dysphonia. Therefore, the mobile-based approach is useful to restore pathological voice and allows for a greater number of patients to easily access voice therapy.

Keywords: Voice disorder, Voice therapy, Mobile-based modality

\section{INTRODUCTION}

Voice disorder is characterized by multi-dimensional aspects resulting from organic, functional, and neurological deficits of vocal mechanisms and can cause voice quality problems such as roughness, breathiness, and strained and tremulous voice [1]. These problems limit participation in daily activities and affect the quality of life of patients [2]. Behavioral, surgical, and medication interventions are widely used to rehabilitate voice quality $[3,4]$. Among these is voice therapy (VT), a behavioral intervention that is the best treatment for use by speech language pathologists (SLPs) $[5,6]$.

The ultimate goal of VT is to restore vocal function to reduce misuse, abuse, and trauma in daily life [7]. VT is the primary option for benign vocal fold lesions and functional dysphonia because it targets voice problems and prevents recurrence in most patients with dysphonia. Moreover, VT prevents serious complications associated with

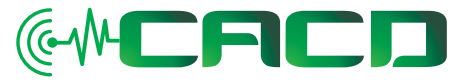

Received: September 21, 2020 Revision: December 28, 2020 Accepted: April 10, 2021

\section{Correspondence: \\ Soon-Bok Kwon}

Department of Humanities, Language and Information, Pusan National University, 2 Busandaehak-ro 63beongil, Geumjeong-gu, Busan 46241, Korea Tel: +82-51-510-2003

Fax: +82-51-582-0572

E-mail: voicesbkwon@gmail.com

(C) 2021 The Korean Association of SpeechLanguage Pathologists

This is an Open Access article distributed under the terms of the Creative Commons Attribution NonCommercial License (https://creativecommons.org/ licenses/by-nc/4.0/) which permits unrestricted noncommercial use, distribution, and reproduction in any medium, provided the original work is properly cited. 
laryngeal microsurgery, including permanent damage such as scarring of the vocal folds. Even if such damage has occurred, VT in many cases can be performed to produce a good voice. In addition, robust acoustic parameters were introduced for voice quality evaluation. Pathological voice tends to decrease the score of cepstral peak prominence (CPP), smoothed cepstral peak prominence (CPPS) and increase the scores of acoustic voice quality index (AVQI) and acoustic breathiness index (ABI) [8-13].

Visual and auditory feedback is important for VT. During the session, the patient is motivated by visually confirming the voice's tone, intensity, and speed, and listening to and comparing the voice before and after the treatment. The auditory feedback provides information on the physiological and functional vocal conditions that can affect the voice quality, and the visual feedback can confirm whether the vocalization is correct according to the clinician's modeling. It has been reported that visual and auditory feedback is effective not only for patients with voice disorders, but also for patients with hearing impairment, neuro-linguistic disorders, fluency disorders, and articulation disorders [14-16].

The most widely used visual feedback method in VT is to use the real-time pitch of the Computerized Speech Lab (CSL; KayPentax, model 4500), Dr. Speech, version 4 (DR. Speech4), Wavesurfer, and Speech Analyzer [17-21]. These programs can show the modeling of the phonation to patients and provide repeated listening of the voice. This visual and auditory model allows the patient to attempt to produce speech in an appropriate manner. However, this CSL program is expensive and difficult to use in speech-language clinics. Thus, there is a need for an easier and more convenient way to provide similar visual and auditory feedback to patients in VT.

Recently, the development of mobile apps has led to the creation of apps that can assess voice quality, frequency, and intensity in real-time. 'Voice Test' for voice quality analysis, 'Voice Tool' and 'Voice analyst' for visual feedback of pitch and loudness, 'Metronome' for rhythm synchronization, 'PaTaKa DDK test' for diadochokinesis, 'VoCo' for vocal training, 'Vocal folds Nodules Flying' for voice game, and 'RBH Learning' for auditory-perceptual training. The visual display of the frequency and intensity of the voice by the apps can still be used in VT.

The purpose of this study was to verify the effectiveness of mobile-based voice therapy (MBVT) compared to existing traditional treatment methods. We randomly classified patients with various speech disorders as receiving MBVT or tradi- tional voice therapy (TRVT) and assessed the effectiveness of each type of VT.

\section{METHODS}

\section{Subjects}

A total of 45 individuals participated in this study. The subjects were recruited from one hospital in Busan, South Korea. We included subjects with benign vocal fold lesions who exhibited dysphonia at varying levels of severity. The medical diagnosis was determined by an otolaryngologist based on the patient's medical chart, patient interview, and laryngeal videoendoscopy and stroboscopy results. The initial study population consisted of 48 subjects who provided negative samples, three of whom were excluded after the signal-tonoise ratio (SNR) analysis because the samples did not meet the SNR criteria of at least $30 \mathrm{~dB}$. SNR below $30 \mathrm{~dB}$ does not guarantee reliable voice analysis. The subjects included 19 men and 26 women with a mean age ( \pm standard deviation [SD]) of $46.3 \pm 10.5$ years (range: $32-67$ years) (Table 1).

\section{Measurements}

\section{Acoustic assessment}

Prior to each VT session, voice samples from the subject were recorded by CSL. The recordings included the patient (1) producing the sustained vowel /a/ phonation at a comfortable and habitual pitch and loudness three times, and (2) reading one Korean sentence from the "Walk" passage with 26 syllables. Acoustic assessments were carried out through three types of tests: cepstral analysis, AVQI version 2 (AVQIv2) and 3 (AVQIv3), and ABI. Cepstral analysis outputs two variables, CPP and CPPS. AVQI and ABI are multivariate models that quantify pathological voices [8,9]. The Institutional Review Board of Pusan National University Hospital approved this

Table 1. Description of AR and TR groups

\begin{tabular}{lcc}
\hline Variables & MBVT (N=25) & TRVT (N=20) \\
\hline Age & $49.4 \pm 10.3$ & $43.7 \pm 11.2$ \\
R/0 & 13 & 12 \\
Vocal fold nodules & 12 & 8 \\
Vocal fold polyp & 10 & 9 \\
Gender & 15 & 11 \\
Male & & \\
Female & 15 & \\
\hline
\end{tabular}

MBVT, mobile-based voice therapy; TRVT, traditional voice therapy; R/O, rule out. 
study (H-1801012063).

\section{Auditory-perceptual (A-P) ratings}

The samples for A-P ratings were randomly selected, and the voice severity was evaluated by three SLPs. A-P ratings were performed using the Grade, Roughness, Breathiness, Asthenia, Strain (GRBAS) scale [22], and the Consensus AuditoryPerceptual Evaluation of Voice (CAPE-V) [23]. The GRBAS scale is based on a four-point Likert scale (normal: 0; mild: 1 ; moderate: 2; and severe: 3 ), and the CAPE-V was based on the visual analog scale of $100 \mathrm{~mm}$, with anchoring points (normal: 0 , severe: 100). For each rating, the grade (G) from the GRBAS and overall severity (OS) from the CAPE-V were measured as an indication of voice severity. $G$ and OS are variables that indicate the overall severity of voice, and are generally known to have the highest reliability among several auditory-perceptual variables.

\section{Self-rated questionnaire}

The Voice Handicap Index 10 (VHI-10) is a patient-based selfassessment tool consisting of 10 items [24]. The VHI-10 is based on a five-point Likert scale (from $0=$ never to $4=$ always) and assesses three domains: functional, physical, and emotional aspects of voice disorders. The overall aim of the VHI10 is to quantify the patient's perception of their own voice handicap. The VHI-10 can evaluate the effectiveness and satisfaction of intervention and follow-up after VT, medication, and surgical intervention. It is a good indicator of patient satisfaction with the intervention [24].

\section{Procedures}

Forty-five subjects were included according to the study criteria, and the VT procedures were explained to the subjects. To compare the voice quality, voice assessments were performed before and after VT (after 8 weeks). After the first voice assessment to check their voice quality grade, subjects were randomly assigned to either an experimental group for MBVT, or a control group for TRVT. Both groups participated in training sessions for 40 minutes a day, 1 day per week, over a period of 8 weeks, for a total of 8 sessions.

There were four VT techniques that were implemented in the study and are widely used in VT: vocal function exercise, accent method, inhalation phonation, and semi-occluded vocal tract exercises [25-27]. The VT included four different techniques that subjects performed for 10 minutes each, in a circular manner. Each technique was based on three levels of
Table 2. Voice therapy procedure

\begin{tabular}{|c|c|c|c|c|}
\hline tep & VT & TRVT & & lıme \\
\hline & \multirow{4}{*}{$\begin{array}{c}\text { Using } \\
\text { Voice Analyst, } \\
\text { Pro } \\
\text { Metronome, } \\
\text { and VoCo on } \\
\text { tablet PC }\end{array}$} & \multirow{4}{*}{$\begin{array}{c}\text { Using } \\
\text { Real-time pitch } \\
\text { of the } \\
\text { Computerized } \\
\text { Speech Lab }\end{array}$} & exer & \\
\hline tep 2 & & & Acce & 10 \\
\hline ten & & & Inhala & 10 \\
\hline $\operatorname{lop} 4$ & & & $\begin{array}{l}\text { Semi-occluded vocal } \\
\text { tract exercises }\end{array}$ & \\
\hline
\end{tabular}

difficulty, and the SLP provided appropriate techniques for the subject's ability level (Table 2).

Both groups performed the same VT procedure. The difference was what software was used during the VT. The TRVT used the existing real-time pitch program in the CSL and a manual metronome. The MBVT used three Android apps: Voice Analyst (Speechtools Ltd), Pro Metronome (EUMLab), and VoCo (SEAVIEW MEDIA Ltd) on tablet PC (Galaxy Tab S5e 10.5", Samsung Electronics).

\section{Statistics}

All statistical analyses were completed using $\mathrm{R}$ version 3.4.1 (The R Foundation for Statistical Computing, Vienna, Austria) and RStudio 1.0.143 (RStudio Inc., Boston, MA, USA). An independent t-test was performed to compare the voice evaluation of both the MBVT and TRVT groups, and a paired t-test was used to compare pre- and post-VT for both groups. The statistical significance level was set at $\alpha=0.05$.

The Spearman rank-order correlation coefficients $\left(\mathrm{r}_{\mathrm{s}}\right)$ were measured among acoustic analysis, A-P ratings, and the selfrated questionnaire. The correlation coefficient $[r]$ was classified as follows: $r \geq 0.9$, very high; $0.7 \leq r<0.9$, high; $0.5 \leq r<0.7$, moderate; $0.3 \leq r<0.5$, low; and $r<0.3$, negligible [28].

\section{RESULTS}

\section{Changes after MBVT and TRVT}

Table 3 shows the paired t-test results measured before and after the MBVT and TRVT for all variables. For both types of VT, there were statistically significant changes in all variables $(p<0.01)$. It was confirmed that the measured values of CPP and CPPS increased, and the values of AVQI, ABI, Grade, OS, and VHI-10 decreased. Based on these measurements, it was confirmed that the voice quality improved after VT.

\section{Comparison of voice measures between MBVT and TRVT}

There were no differences between the two groups (MBVT 
Table 3. Paired t-test of before and after MBVT and TRVT

\begin{tabular}{|c|c|c|c|c|c|c|c|c|}
\hline & & & & & & & & \\
\hline & Pre & Post & $t$ & $p$ & Pre & Post & $t$ & $p$ \\
\hline СРР & $18.2 \pm 2.0$ & $21.1 \pm 2.9$ & -5.986 & $<0.01^{* *}$ & $17.8 \pm 3.0$ & $20.1 \pm 2.4$ & -4.685 & $<0.01^{* *}$ \\
\hline CPPS & $9.4 \pm 1.4$ & $11.5 \pm 1.9$ & -5.823 & $<0.01^{* *}$ & $9.4 \pm 2.3$ & $11.1 \pm 1.7$ & -5.505 & $<0.01^{* *}$ \\
\hline AVQlv2 & $6.2 \pm 0.8$ & $5.3 \pm 1.0$ & 4.467 & $<0.01^{* *}$ & $6.3 \pm 1.1$ & $5.4 \pm 0.8$ & 4.934 & $<0.01^{* *}$ \\
\hline AVQlv3 & $4.9 \pm 1.1$ & $3.5 \pm 1.2$ & 5.566 & $<0.01^{* *}$ & $5.3 \pm 1.6$ & $3.8 \pm 1.1$ & 7.082 & $<0.01^{* *}$ \\
\hline $\mathrm{ABI}$ & $4.7 \pm 1.4$ & $3.4 \pm 1.3$ & 4.711 & $<0.01^{* *}$ & $5.2 \pm 1.1$ & $4.0 \pm 1.1$ & 4.445 & $<0.01^{* *}$ \\
\hline Grade & $1.9 \pm 0.5$ & $0.6 \pm 0.4$ & 13.966 & $<0.01^{* *}$ & $2.1 \pm 0.6$ & $0.8 \pm 0.4$ & 13.516 & $<0.01^{* *}$ \\
\hline OS & $51.1 \pm 15.8$ & $22.8 \pm 12.6$ & 8.458 & $<0.01^{* *}$ & $56.1 \pm 15.0$ & $24.2 \pm 11.6$ & 12.310 & $<0.01^{* *}$ \\
\hline VHI-10 & $19.5 \pm 4.9$ & $15.7 \pm 4.5$ & 5.818 & $<0.01^{* *}$ & $20.8 \pm 5.2$ & $16.2 \pm 3.6$ & 7.579 & $<0.01^{* *}$ \\
\hline
\end{tabular}

${ }^{* *} p<0.01$.

Table 4. Independent t-test between MBVT and TRVT

\begin{tabular}{|c|c|c|c|c|c|c|c|c|}
\hline & \multicolumn{4}{|c|}{ Pre } & \multicolumn{4}{|c|}{ Post } \\
\hline & MBVT & TRVT & $t$ & $p$ & MBVT & TRVT & $t$ & $p$ \\
\hline СРP & $18.2 \pm 2.0$ & $17.8 \pm 3.0$ & 0.760 & 0.453 & $21.1 \pm 2.9$ & $20.1 \pm 2.4$ & 1.457 & 0.086 \\
\hline CPPS & $9.4 \pm 1.4$ & $9.4 \pm 2.3$ & 0.193 & 0.846 & $11.5 \pm 1.9$ & $11.1 \pm 1.7$ & 1.344 & 0.246 \\
\hline AVQlv2 & $6.2 \pm 0.8$ & $6.3 \pm 1.1$ & -0.379 & 0.707 & $5.3 \pm 1.0$ & $5.4 \pm 0.8$ & -0.591 & 0.327 \\
\hline AVQIv3 & $4.9 \pm 1.1$ & $5.3 \pm 1.6$ & -1.283 & 0.206 & $3.5 \pm 1.2$ & $3.8 \pm 1.1$ & -1.357 & 0.121 \\
\hline $\mathrm{ABI}$ & $4.7 \pm 1.4$ & $5.2 \pm 1.1$ & -1.725 & 0.092 & $3.4 \pm 1.3$ & $4.0 \pm 1.1$ & -2.591 & $0.013^{*}$ \\
\hline Grade & $1.9 \pm 0.5$ & $2.0 \pm 0.6$ & -1.269 & 0.211 & $0.6 \pm 0.4$ & $0.8 \pm 0.4$ & -2.406 & $0.021^{*}$ \\
\hline OS & $51.1 \pm 15.8$ & $56.1 \pm 15.0$ & -1.607 & 0.115 & $22.8 \pm 12.6$ & $24.2 \pm 11.6$ & -0.869 & 0.389 \\
\hline VHI-10 & $19.5 \pm 4.9$ & $20.8 \pm 5.2$ & -1.094 & 0.280 & $15.7 \pm 4.5$ & $16.2 \pm 3.6$ & -0.849 & 0.400 \\
\hline
\end{tabular}

${ }^{*} p<0.05$.

Table 5. Correlations among acoustic analysis, A-P ratings, and the self-rated questionnaire

\begin{tabular}{|c|c|c|c|c|c|c|c|c|}
\hline & CPP & CPPS & AVQIv2 & AVQIv3 & $A B I$ & Grade & OS & VHI10 \\
\hline СРР & 1 & $.954^{* *}$ & $-.822^{* *}$ & $-.891^{* *}$ & $-.786^{* *}$ & $-.789^{* *}$ & $-.771^{* *}$ & $-.797^{* *}$ \\
\hline CPPS & & 1 & $-.910^{* *}$ & $-.934^{* *}$ & $-.701^{* *}$ & $-.803^{* *}$ & $-.784^{* *}$ & $-.826^{* *}$ \\
\hline AVQlv2 & & & 1 & $.932^{* *}$ & $.621^{* *}$ & $.770^{* *}$ & $.794^{* *}$ & $.817^{* *}$ \\
\hline AVQIv3 & & & & 1 & $.796^{* *}$ & $.860^{* *}$ & $.858^{* *}$ & $.853^{* *}$ \\
\hline$A B I$ & & & & & 1 & $.784^{* *}$ & $.775^{* *}$ & $.687^{* *}$ \\
\hline Grade & & & & & & 1 & $.917^{* *}$ & $.759^{* *}$ \\
\hline OS & & & & & & & 1 & $.746^{* *}$ \\
\hline VHI10 & & & & & & & & 1 \\
\hline
\end{tabular}

${ }^{* *} p<0.01$.

and TRVT) in the pre-treatment measurements $(0.092<p$ $<0.846)$. After treatment, we found statistically significant differences in two (ABI, $p=0.013$ and Grade, $p=0.021$ ) of the eight variables between the two groups. The ABI and Grade scores of the MBVT group were lower than those of the TRVT group, indicating greater voice recovery in the MBVT group
(Table 4).

Correlations among acoustic analysis, A-P ratings, and the self-rated questionnaire

The correlations among acoustic analyses (CPP, CPPS, AVQIv2, AVQIv3, and ABI), A-P assessments (G and OS), and the self- 
rated questionnaire (VHI-10) are shown in Table 5. The correlations among all variables varied from moderate $(r=0.621$, $p<0.001$, AVQIv2, and ABI) to high $(r=0.954, p<0.001$, CPP and CPPS).

\section{DISCUSSION}

This study was conducted to identify the effects of MBVT on voice quality and vocal functions in patients with voice disorders. The results showed that both MBVT and TRVT can improve the quality and stability of voice, but that MBVT led to an overall higher level of voice recovery than TRVT, as reflected in the ABI and Grade scores.

VT requires patients to make extensive behavioral changes between VT sessions and to maintain these changes after discharge or in the home [29]. VT involves vocal motor learning, [30] or the acquisition and generalization of a target voice production technique. In addition to motor learning, VT requires behavioral self-regulation, volitional goal-directed planning, and the execution, self-evaluation, and self-correction of this technique [31].

Digital technology is commonly used in our lives, and can be used in various forms, such as application software, virtual reality, the Internet of Things, and big data depending on the intention of users. Among them, most apps are technology that uses smart devices and can be considered to be the most accessible digital technology in a situation where the penetration rate of smartphones is increasing [32]. Apps are actively being created in other fields due to ease of development and user convenience, but the development of pertinent apps in the field of speech language pathology is rare [33]. In addition, most SLPs have smart devices, but they rarely use smart contents in speech-language therapy sessions [34]. Smart content is effective for treatment, but is difficult to use because of the lack of various developed content [35]. For example, for speech therapy in general, two dimensional (2D) picture cards for nouns, verbs, adjectives, etc. are created, and if necessary, three dimensional (3D) video production is also carried out. A major limitation is the speed by which content can be created for all of the words that are used. In the future, it will be necessary to allow users to directly upload pictures or videos to expand the available content.

Although there has been a lack of smart content that can be used in clinical practice, research using information technologies, such as smartphone-based apps has been reported in the field of speech language pathology in Korea. Jeong [36] re- ported that oral mobility, speech intelligibility, phrase length, and overall speech-language skills were improved in school aged children with cerebral palsy through the use of a smartphone app for speech language therapy. In another study, a smartphone-based VT and management app was developed for patients with vocal fold nodules. The VT app consisted of blowing, and word and sentence training. It was found that users were generally satisfied with the app [37]. It has been reported that speech language therapy using a smart device app has had positive results in improving speech-language ability in various communication disorders, such as language development disorder and neuro-lingual disorder. However, there are few studies on apps and app development for voice disorders [38].

That said, VT research using information technology has been actively conducted in several countries. In one study [39], Fu et al. investigated voice quality and patient satisfaction using VT for vocal nodules through Skype. Through this telepractice, voice quality improved and patient satisfaction of treatment was reported to be high. Telepractice has shown potential as an alternative to face-to-face practice. Verde et al. introduced a mobile app that can estimate fundamental frequency (F0) and perform brief pathological voice screening [38]. Automatically, the app measures the F0 value and distinguishes pathological from healthy voices to inform the user of their voice status. Mehta et al. reported a smartphone-based voice health monitor that records high-bandwidth accelerometer signals from neck skin [40]. Mahnan developed a noninvasive wearable device that applies vibro-tactile stimulation to laryngeal muscles with the aim to improve voice quality of individuals with spasmodic dysphonia [41]. Van Deer developed an iOS app that calculates and displays CPP values for patient self-monitoring of voice quality [42].

In the present study, patients in the MBVT group showed similar positive improvements in voice recovery and vocal function as those in the TRVT group. Both treatment approaches have been found to be helpful in voice recovery in patients with benign vocal fold lesions. The results of this study confirmed that MBVT could produce similar positive effects compared to conventional PC-based VT. A MBVT setting could be relatively inexpensively constructed compared to the PC-based setting, and has the advantage of being less restricted by the location. In addition, the patient can review the training method they had learned in the clinic at home, which helps maintains a good voice. If the patient's residence is far from the clinic, or in order to continue voice training 
when travel is restricted, the patient can sustain voice training at home through mobile devices. Based on the advantages of using a mobile-based intervention, this study was conducted to confirm the effectiveness of a MBVT method compared to the existing PC-based VT method. If the study design included not only VT but also acoustic and aerodynamic voice evaluation, the PC-based voice evaluation/therapy method is better. However, when limited to VT, good training results can be derived when using MBVT. If a multi-functional VT app, including voice recording, storage, and management of vocal functions, were developed, it would be more widely utilized.

The limitation of this study is that there is a difference in the skill level of handling the device and using the apps. The PCbased system has been used extensively by clinicians, but the mobile-based system may not be as familiar. In addition, updates may occur frequently due to the nature of the app, and technical support may not be available. In preparation for this situation, it is necessary to check several apps in advance.

\section{FINANCIAL DISCLOSURE}

The authors have no funding or financial relationship to disclose

\section{REFERENCES}

1. Roy N, Merrill RM, Thibeault S, Parsa RA, Gray SD, Smith EM. Prevalence of voice disorders in teachers and the general population. J Speech Lang Hear Res. 2004;47:281-293.

2. Sukanen O, Sihvo M, Rorarius E, Lehtihalmes M, Autio V, Kleemola L. Voice Activity and Participation Profile (VAPP) in assessing the effects of voice disorders on patients' quality of life: validity and reliability of the Finnish version of VAPP. Logoped Phoniatr Vocol. 2007;32:3-8.

3. Mattioli F, Bergamini G, Alicandri-Ciufelli Met al. The role of early voice therapy in the incidence of motility recovery in unilateral vocal fold paralysis. Logoped Phoniatr Vocol. 2011;36:40-47.

4. Gallena S, Smith PJ, Zeffiro T, Ludlow CL. Effects of levodopa on laryngeal muscle activity for voice onset and offset in Parkinson disease. J Speech Lang Hear Res. 2001;44:1284-1299.

5. Hillman RE, Gress CD, Hargrave J, Walsh M, Bunting G. The efficacy of speech-language pathology intervention: voice disorders Seminars in Speech and Language: () 1990 by Thieme Medical Publishers, Inc., 1990:297-310.

6. Ruotsalainen JH, Sellman J, Lehto L, Jauhiainen M, Verbeek JH. Interventions for preventing voice disorders in adults. Cochrane Database Syst Rev 2007:CD006372.

7. Salturk Z, Ozdemir E, Sari H, et al. Assessment of Resonant Voice Therapy in the Treatment of Vocal Fold Nodules. J Voice 2019;
33:810 e811-810 e814.

8. Maryn Y, De Bodt M, Roy N. The Acoustic Voice Quality Index: toward improved treatment outcomes assessment in voice disorders. J Commun Disord 2010; 43:161-174.

9. Barsties VLB, Maryn Y, Gerrits E, De Bodt M. The Acoustic Breathiness Index (ABI): A multivariate acoustic model for breathiness. J Voice. 2017;31:511 e511-511 e527.

10. Kim GH, Lee YW, Bae IH, Park HJ, Lee BJ, Kwon SB. Comparison of two versions of the acoustic voice quality index for quantification of dysphonia severity. J Voice. 2020;34:489 e411-489 e419.

11. Awan SN, Roy N, Dromey C. Estimating dysphonia severity in continuous speech: application of a multi-parameter spectral/ cepstral model. Clin Linguist Phon. 2009;23:825-841.

12. Bielamowicz S, Kreiman J, Gerratt BR, Dauer MS, Berke GS. Comparison of voice analysis systems for perturbation measurement. J Speech Hear Res. 1996;39:126-134.

13. Rabinov CR, Kreiman J, Gerratt BR, Bielamowicz S. Comparing reliability of perceptual ratings of roughness and acoustic measure of jitter. J Speech Hear Res. 1995;38:26-32.

14. Ubrig MT, Tsuji RK, Weber R et al. The influence of auditory feedback and vocal rehabilitation on prelingual hearing-impaired individuals post cochlear implant. J Voice. 2019;33:947 e941-947 e949.

15. Jiang Y, Norman KE. Effects of visual and auditory cues on gait initiation in people with Parkinson's disease. Clin Rehabil. 2006;20: 36-45.

16. Chesters J, Baghai-Ravary L, Mottonen R. The effects of delayed auditory and visual feedback on speech production. J Acoust Soc Am. 2015;137:873-883.

17. Chen SH, Hsiao TY, Hsiao LC, Chung YM, Chiang SC. Outcome of resonant voice therapy for female teachers with voice disorders: perceptual, physiological, acoustic, aerodynamic, and functional measurements. J Voice. 2007;21:415-425.

18. Gelfer MP, Tice RM. Perceptual and acoustic outcomes of voice therapy for male-to-female transgender individuals immediately after therapy and 15 months later. J Voice. 2013;27:335-347.

19. Smits I, Ceuppens P, De Bodt MS. A comparative study of acoustic voice measurements by means of dr. speech and computerized speech lab. J Voice. 2005;19:187-196.

20. Sjölander K, Beskow J. Wavesurfer-an open source speech tool Sixth International Conference on Spoken Language Processing. 2000.

21. Godwin-Jones R. Speech tools and technologies. Language Learning \& Technology. 2009;13:4-11.

22. Sakata T, Kubota N, Yonekawa H, Imaizumi S, Niimi S. Grbas evaluation of running speech and sustained phonation. Folia Phoniatr Logop. 1995;47:101-101.

23. Kempster GB, Gerratt BR, Verdolini Abbott K, Barkmeier-Kraemer J, Hillman RE. Consensus auditory-perceptual evaluation of voice: development of a standardized clinical protocol. Am J Speech Lang Pathol. 2009;18:124-132.

24. Rosen CA, Lee AS, Osborne J, Zullo T, Murry T. Development and validation of the voice handicap index-10. Laryngoscope. 2004; 
114:1549-1556.

25. Sauder C, Roy N, Tanner K, Houtz DR, Smith ME. Vocal function exercises for presbylaryngis: a multidimensional assessment of treatment outcomes. Ann Otol Rhinol Laryngol. 2010;119:460467.

26. Bassiouny S. Efficacy of the accent method of voice therapy. Folia Phoniatr Logop 1998; 50:146-164.

27. Manternach JN, Daugherty JF. Effects of a Straw Phonation Protocol on Acoustic and Perceptual Measures of an SATB Chorus. J Voice. 2019;33:80-86.

28. Mukaka MM. Statistics Corner: A guide to appropriate use of Correlation coefficient in medical research. Malawi Med J. 2012; 24:69-71.

29. Speyer R, Wieneke GH, Dejonckere PH. Documentation of progress in voice therapy: perceptual, acoustic, and laryngostroboscopic findings pretherapy and posttherapy. J Voice. 2004;18:325340 .

30. Verdolini K, Lee TD. Optimizing motor learning in speech interventions: Theory and practice. Vocal Rehabilitation for Medical Speech-Language Pathology. 2004:403-446.

31. Carver CS, Scheier MF. On the self-regulation of behavior. Cambridge University Press, 2001.

32. Fan Y, Yang CY. Competition. Product proliferation, and welfare: a study of the US smartphone market. American Economic JournalMicroeconomics. 2020;12:99-134.

33. Lee Y, Lee S, Sung M. Analysis of mobile application trends for speech and language therapy of children with disabilities in Korea. Phonetics and Speech Sciences. 2015;7:153-163.

34. Furlong LM, Morris ME, Erickson S, Serry TA. Quality of mobile phone and tablet mobile apps for speech sound disorders: Proto- col for an evidence-based appraisal. JMIR Res Protoc. 2016; 5:e233.

35. Lee J, Kim Y, You H, Ko B. An analysis of parents' and experts' needs for smart content for speech and language therapy support for people with speech disorders. J Speech Hear Disord. 2015; 24:171-182.

36. Jeong P. Speech-language therapy using apps for a student with cerebral palsy. AAC Research \& Practice. 2014;2:119-126.

37. Kim J, Kim Y, Park J, et al. Evaluation of a smartphone application on vocal rehabilitation in patients with vocal nodules. Proceedings of 7th KIEE. 2017:23-25.

38. Verde L, De Pietro G, Veltri P, Sannino G. An m-health system for the estimation of voice disorders 2015 IEEE International Conference on Multimedia \& Expo Workshops (ICMEW): IEEE, 2015:16.

39. Fu S, Theodoros DG, Ward EC. Delivery of intensive voice therapy for vocal fold nodules via telepractice: A pilot feasibility and efficacy study. J Voice. 2015;29:696-706.

40. Mehta DD, Zañartu M, Van Stan JH, Feng SW, Cheyne HA, Hillman RE. Smartphone-based detection of voice disorders by longterm monitoring of neck acceleration features 2013 IEEE International Conference on Body Sensor Networks: IEEE, 2013:1-6.

41. Mahnan A, Konczak J, Faraji SA. Wearable non-invasive neuromodulation device for the symptomatic treatment of the voice disorder spasmodic dysphonia 2019 Design of Medical Devices Conference: American Society of Mechanical Engineers Digital Collection, 2019.

42. van Leer E, Pfister RC, Zhou X. An iOS-based cepstral peak prominence application: Feasibility for patient practice of resonant voice. J Voice. 2017;31:131 e139-131 e116. 